Research Article

\title{
Groundwater Depth Forecasting Using a Coupled Model
}

\author{
Manfei Zhang $\mathbb{D},{ }^{1,2}$ Yimeng Wang, ${ }^{1,2}$ Xiao Wang, ${ }^{1,2}$ and Weibo Zhou $\mathbb{D}^{1,2}$ \\ ${ }^{1}$ School of Water and Environment, Chang'an University, Xi'an 710054, China \\ ${ }^{2}$ Key Laboratory of Subsurface Hydrology and Ecological Effects in Arid Region, Chang'an University, Xi'an 710054, China
}

Correspondence should be addressed to Weibo Zhou; zhouweibopro@163.com

Received 3 November 2020; Revised 10 January 2021; Accepted 29 January 2021; Published 24 February 2021

Academic Editor: Rodica Luca

Copyright $(2021$ Manfei Zhang et al. This is an open access article distributed under the Creative Commons Attribution License, which permits unrestricted use, distribution, and reproduction in any medium, provided the original work is properly cited.

Accurate and reliable prediction of groundwater depth is a critical component in water resources management. In this paper, a new method based on coupling wavelet decomposition method (WA), autoregressive moving average (ARMA) model, and BP neural network (BP) model for groundwater depth forecasting applications was proposed. The relative performance of the proposed coupled model (WA-ARMA-BP) was compared to the regular autoregressive integrated moving average (ARIMA) and $\mathrm{BP}$ models for annual average groundwater depth forecasting using leave-one-out cross-validation (LOO-CV). The variables used to develop and validate the models were average groundwater depth data recorded from 1981 to 2010 in Jinghui Canal Irrigation District in the northwest of China. It was found that the WA-ARMA-BP model provided more accurate annual average groundwater depth forecasts compared to the ARIMA and BP models. The results of the study indicate the potential of the WAARMA-BP model in forecasting nonstationary time series such as groundwater depth.

\section{Introduction}

Groundwater is a major source of water supply for industry and agriculture in many countries. However, with the development of urbanization and augment of water consumption, groundwater has been overexploited which leads to harmful environmental side effects, for example, major water-level declines, drying up of wells, reduction of water in streams and lakes, water-quality degradation, increased pumping costs, land subsidence, and decreased well yields, in many parts of the word, especially in developing countries $[1,2]$. In order to prevent the above situation and manage groundwater resources more effectively, accurate and reliable forecasting of groundwater level (GWL) is crucial [3]. However, the GWL is very complex and highly nonlinear as it depends on many complex factors such as precipitation and temperature. Therefore, developing effective models that can predict GWL precisely is imperative [4].

Physical-based numerical models have been frequently employed for the simulation of the GWL $[5,6]$. However, to develop a physical-based numerical model needs collecting a great deal of data such as thickness and hydraulic properties of each hydrogeological unit, groundwater recharge/discharge, topography information, geological coverage, soil properties, land use map, vegetation distribution, evapotranspiration information, and hydrologic and climatic data [7]. A large quantity of precise data/parameters of study domain are required in the calibration and validation process of the model [8]. In addition, because numerical models make many assumptions on the governing equation in porous media and most of the model forcings such as evapotranspiration and rainfall are less predictable, they are less competent in forecasting. Therefore, the use of physicalbased models is highly restricted.

Statistical models are a suitable alternative when data is limited and obtaining accurate forecasts is more important than conceiving underlying mechanisms [9]. For example, Bidwell (2005) [10] developed an autoregressive moving average (ARMA) model to predict GWL in New Zealand. Lee et al. (2009) [11] used an ARMA model to forecast the GWL in Changwon, Korea. Guzman et al. (2017) [12] applied an ANN model to simulate daily GWL at a local scale in the Mississippi River Valley Alluvial Aquifer, located in the southeastern United States. Yaseen et al. (2019) [13] applied 
the bioinspired adaptive neurofuzzy inference system (ANFIS) models to forecast the highly nonlinear streamflow of the Pahang River.

However, the above models frequently have limitations with nonstationary data and cannot handle nonstationary data without input data preprocessing [14]. The methods for dealing with nonstationary data are not as advanced as those for stationary data and additional research is needed to investigate methods that are better able to handle nonstationary data effectively. An example of such a method is wavelet analysis.

Preliminary studies have indicated that wavelet analysis (WA) is more effective when analyzing nonstationary time series. WA can decompose an observed time series variables (such as GWL) into various components so that the newly established time series can be used as inputs for an ANN model [15-17]. In recent years, coupled wavelet-artificial neural network (WA-ANN) models have been widely used for the prediction and forecasting in hydrological studies. For instance, Wang et al. (2009) [18] developed a wavelet neural network model to forecast the inflow at the Three Gorges Dam in Yangtze River. Adamowski and Chan (2011) [2] proposed WA-ANN models for 1-month-ahead forecasting of GWL at two sites in the Chateauguay watershed in Quebec, Canada. It was found that WA-ANN models provide more accurate forecasts compared to the ANN and ARIMA models.

In this research, the groundwater depth series was decomposed into multiple detailed signal sequences and approximate signal sequences through wavelet decomposition and reconstruction. Then, detailed signal sequences and approximate signal sequences were calculated and predicted using the ARMA model and BP neural network model, respectively. The coupled model mentioned above is called the WA-ARMA-BP model. The objective of this paper was to develop a WA-ARMA-BP model and compare it with the ARIMA model and the BP neural network model for the forecast of groundwater depth in Jinghui Canal Irrigation District in the northwest of China.

1.1. Study Area and Data Description. The Jinghui Canal Irrigation District lies at a longitude of $108^{\circ} 34^{\prime} 34^{\prime \prime}$ to $109^{\circ} 21^{\prime} 35^{\prime \prime} \mathrm{N}$ and latitude of $34^{\circ} 25^{\prime} 20^{\prime \prime}$ to $34^{\circ} 41^{\prime} 40^{\prime \prime} \mathrm{E}$ in Shaanxi Province, China (Figure 1). It covers an area of $1180 \mathrm{~km}^{2}$, with a continental semiarid climate. The average annual temperature is approximately $13.4^{\circ} \mathrm{C}$. The average annual precipitation is about $538.9 \mathrm{~mm}$, most of which occur during May and October. Rural population accounts for approximately $84 \%$ of the total population, and cultivated land accounts for approximately $80 \%$ of the total area.

The irrigation water resources of Jinghui Canal Irrigation District are Jing River and groundwater. Recently, with the intensification of industrial and agricultural activities, the demand for water resources is constantly increasing. At the same time, affected by the decrease of water inflow from Jing River and the expansion of the scale of motor-operated wells, the water consumption ratio of well and canal irrigation has risen from 0.3 in the 1970 s to 1.3 at present. The increase in the amount of groundwater exploitation results in the increase of groundwater depth in the irrigated areas. Over the course of the recent decades, the increasing groundwater depth has caused a series of ecological and environmental problems in Jinghui Canal Irrigation District, for example, land subsidence and soil salinization [19]. Thus, accurate predictions of GWL fluctuations would be imperative for water resources management in this area.

As groundwater cannot be renewed artificially on a large scale, sustainable management of this resource is vital and its nonsustainable use is a serious danger. It needs a globally averaged analysis of the groundwater depth [7]. To calculate the average groundwater depth over the study area, observed groundwater depth data from 1981 to 2010 from 15 observation wells were obtained from Shaanxi Jinghui Canal Irrigation Administration. Figure 2 presents the locations of the observation wells across the study area. Descriptive statistics of the average groundwater depth in the Jinghui Canal Irrigation District (1981 to 2010) are listed in Table 1. The average groundwater depth varies from $3.98 \mathrm{~m}$ in 1981 to $14.29 \mathrm{~m}$ in 2010 . The average increase rate is $0.34 \mathrm{~m} /$ year.

\section{Methodology}

2.1. Wavelet Decomposition Method (WA). The main feature of the wavelet decomposition method is to decompose the nonstationary time series into those which are much more stationary than the original time series and then study each sequence separately to realize the simulation and prediction of the nonstationary time series. Mallat algorithm [20] is generally used for multiscale decomposition and reconstruction. Actually, the time series $f(t)$ is decomposed based on different frequency channel components in the Mallat algorithm. The time series $f(t)$ can be described as

$$
f(t)=\sum_{k} c_{k}^{0} \phi_{0 k}
$$

The $n$th wavelet decomposition of $f(t)$ can be expressed as

$$
\begin{aligned}
f(t) & =R_{1} f+R_{2} f+\cdots+R_{n} f+P_{n} f, \\
P_{n} f & =\sum_{k} c_{k}^{n} \phi_{n k}, \\
R_{n} f & =P_{n-1} f-P_{n} f=\sum_{k} d_{k}^{n} \psi_{n k}, \\
c^{j} & =H c^{j-1}, \\
d^{j} & =G c^{j-1},
\end{aligned}
$$

where $c^{j}$ and $d^{j}$ represent the approximation signal and detail signal of the original signal $c^{0}$ at $2^{-j}$ resolution, respectively; $H$ is the low pass filter; $G$ is high pass filter; $\varphi_{n k}$ and $\psi_{n k}$ represent the orthogonal basis; and $P_{0} f=f$.

In this way, the nonstationary time series $f(t)$ is decomposed into the superposition form of $n$ detailed signal sequences $R_{1} f, R_{2} f, \ldots R_{n} f$ and 1 approximation signal sequence $P_{n} f$.

Since the number of signal points of the approximation signal and detail signal obtained by the Mallat algorithm is 

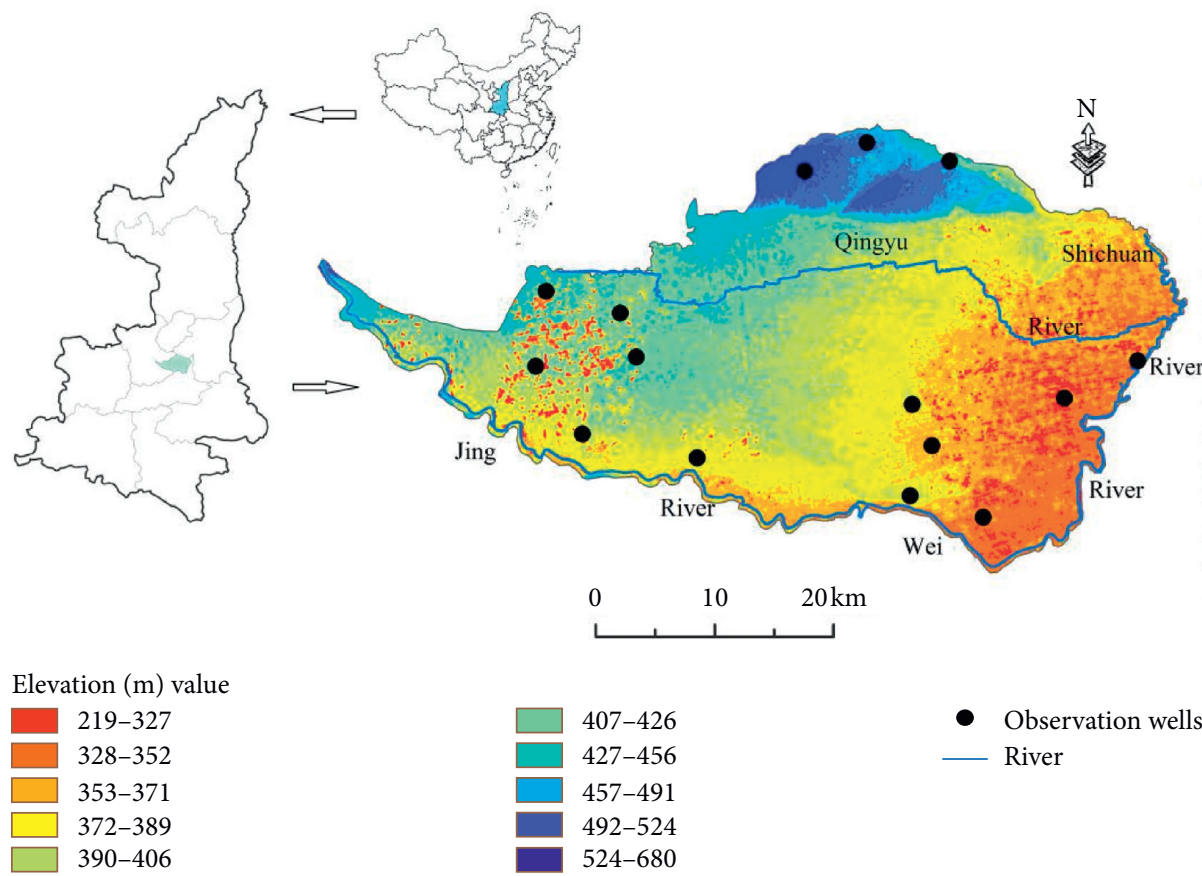

FIgURE 1: Location map of the study area.

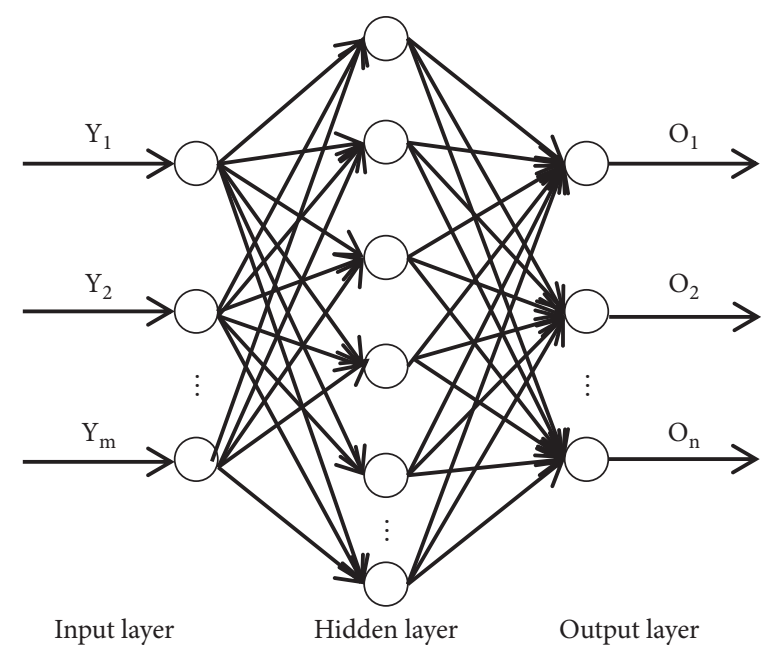

FIgURE 2: Topology structure of BP neural network.

Table 1: Descriptive statistics of the average groundwater depth in the Jinghui Canal Irrigation District (1981 to 2010).

\begin{tabular}{lcccc}
\hline & Minimum & Mean & Maximum & Standard deviation \\
\hline Average groundwater depth $(\mathrm{m})$ & 3.48 & 9.98 & 14.67 & 3.89 \\
\hline
\end{tabular}

less than the number of signal points of the original signal, the decomposed signals can be reconstructed by reconstruction algorithm as follows:

$$
\begin{aligned}
P_{n-1} f & =P_{n} f+R_{n} f, \\
c^{j-1} & =H^{*} c^{j}+G^{*} d^{j},
\end{aligned}
$$

where $H^{*}$ and $G^{*}$ denote the dual operators of $H$ and $G$, respectively.
2.2. ARMA and ARIMA Models. Autoregressive moving average (ARMA) and autoregressive integrated moving average (ARIMA) models are the most commonly used stochastic models.

In stochastic modeling techniques, data should be divided into trend, periodical, autoregressive, and random components. If the statistical characteristics have increasing or decreasing changes over time, there will be a trend term; if they change periodically, there will be a periodic term; and if 
a large change occurs suddenly and it continues, there will be a jump term. The three parts of the trend, period, and jump in time series are deterministic and predictable components that should be omitted for modeling with stochastic methods, and the remaining term is the stochastic term which is used for modeling [21].

The average groundwater depth of the study area from 1981 to 2010 experiences obvious increasing changes over time, which is clear by observing the time series of the data (Figure 3). There were neither periodic fluctuations nor suddenly major changes in the data series, which indicated that the trend term and jump term did not exist.

The Mann-Kendall nonparametric test is widely applied in hydroclimatic trend analysis. However, the performance of the Mann-Kendall (MK) method is affected by the presence of autocorrelation [22-24]. Therefore, a modified Mann-Kendall (MMK) test introduced by Hamed and Rao (1998) [22] was employed to evaluate the trend of serial correlation data. The relationship of the traditional $\mathrm{MK}$ nonparametric test is as follows $[25,26]$ :

$$
\begin{aligned}
U_{M K} & = \begin{cases}\frac{M K-1}{\sqrt{\operatorname{Var}(M K)}}, & M K>0, \\
0, & M K=0, \\
\frac{M K+1}{\sqrt{\operatorname{Var}(M K)}}, & M K<0,\end{cases} \\
M K & =\sum_{i=1}^{N-1} \sum_{j=i+1}^{N} \operatorname{sgn}\left(x_{j}-x_{i}\right), \\
\operatorname{Var}(M K) & =\frac{N(N-1)(2 N+5),}{18},
\end{aligned}
$$

where $U_{M K}$ is the standard of Mann-Kendall statistic; $M K$ is the Mann-Kendall statistic; $\operatorname{Var}(M K)$ is the variance of $M K$; sgn is the sign function; and $N$ is the number of samples. A positive $M K$ value indicates an increasing trend, while a negative $M K$ value shows a decreasing trend. In this paper, the significance level of $5 \%$ is adopted. If $\left|U_{M K}\right|>1.96$, the trend is obvious.

In MMK test, $\operatorname{Var}(M K)^{*}$ is used as the corrected variance [22].

$$
\begin{aligned}
\operatorname{Var}(M K)^{*} & =\frac{n}{n^{*}} \bullet \operatorname{Var}(M K), \\
\frac{n}{n^{*}} & =1+\frac{2}{n(n-1)(n-2)} \sum_{i=1}^{n-1}(n-i)(n-i-1) \\
(n-i-2) r_{i}, &
\end{aligned}
$$

where $n$ is the actual number of observations, $n^{*}$ is the effective sample size, $r_{i}$ is the lag $i$ significant autocorrelation coefficient of $i$ th rank of time series, and its expression is

$$
r_{i}=\frac{(1 / n-i) \sum_{j=1}^{n-j}\left(x_{j}-\bar{x}\right)\left(x_{i+j}-\bar{x}\right)}{(1 / n) \sum_{i=1}^{n}\left(x_{i}-\bar{x}\right)^{2}}
$$

Statistic $U_{M K}^{*}$ of MMK trend test method is computed by replacing $\operatorname{Var}(M K)$ in equation (4) with $\operatorname{Var}(M K)^{*}$. The rule used to determine the trend and significance of $U_{M K}^{*}$ is similar to $U_{M K}$.

If the MMK test results indicate nonstationary factors, it is necessary to remove the trend term. Differencing is also one of the most basic methods of data stationary. In this method, each data is reduced from the previous data, and the differentiated series $(\operatorname{Diff}(t)=T(t)-T(t-1))$ is obtained. The stationary time series' Auto Correlation Function (ACF) structure can be detected by drawing a correlogram. If the graph damps down to zero rapidly in exponential or oscillatory form, the time series is stationary.

ARMA model provides a parsimonious description of a weakly stationary stochastic process. The ARMA model is developed via combining the AR (autoregressive) and MA (moving average) models. ARMA (p, q) models are defined as [27]

$$
x_{t}=\sum_{i=1}^{p} \phi_{i} x_{t-i}+\sum_{j=1}^{q} \theta_{j} w_{t-j}+w_{t},
$$

where $x_{t}(t=0, \pm 1, \pm 2, \ldots)$ represents a time series; $\varphi_{1}$, $\varphi_{2}, \ldots, \varphi_{p}$ and $\theta_{1}, \theta_{2}, \ldots, \theta_{q}$ are called the AR and MA parameters, respectively; the parameter $p$ and $q$ denote the AR and MA orders, respectively; $w_{t}(t=0, \pm 1, \pm 2, \ldots)$ is a Gaussian white noise sequence.

When a time series does not appear to be covariance stationary, the differencing procedure may be applied to make it stationary. The ARMA (p, q) model can be applied to the stationary differenced time series and the model so constructed is called the ARIMA (p, $d, \mathrm{q})$ model where $d$ denotes the order of differencing.

ARIMA modeling involves three stages: identification stage, estimation stage, and diagnostic checking stage.

Firstly, in the identification stage, it is ensured whether the time series is stationary or not. For nonstationary time series data, a differencing process is applied till the data series are transformed into stationary ones. Auto Correlation Functions (ACFs) and Partial Auto Correlation Functions (PACFs) are commonly used in evaluating a time series variable's dependency on its past. The AR and MA terms of the stationary time series are determined by examining the patterns of the graphs of ACFs and PACFs. Autocorrelation is the correlation between time series and the same time series lag, while partial autocorrelations are the correlation coefficients between the basic time series and the same time series lag, but with the elimination of the influence of the members in between [28].

Secondly, in the estimation stage, parameters $p$ and $q$ of $\mathrm{AR}$ and MA terms are estimated. Parameters $p$ and $q$ can be identified by looking at ACF, which is a bar chart of time series of coefficients of correlation of time series and lags of itself, and PACF, which represents the plot of partial 


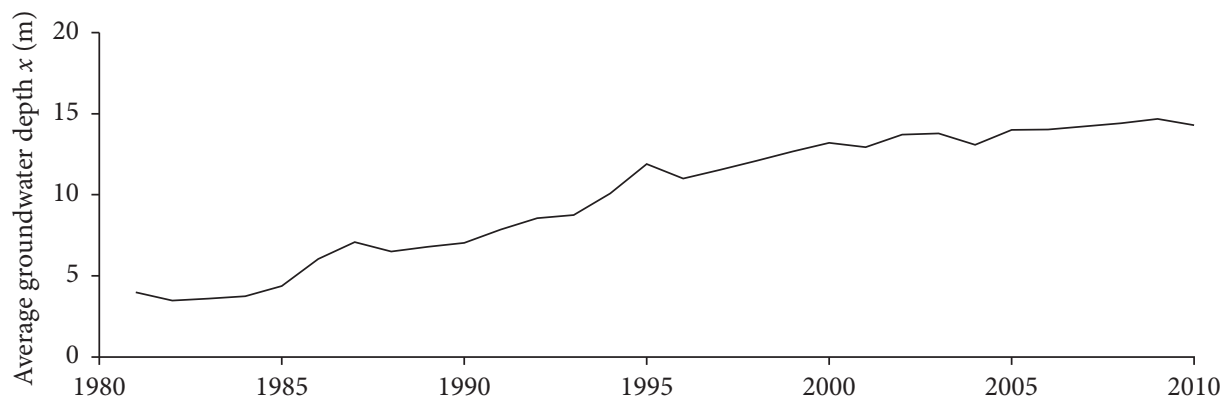

Figure 3: The average groundwater depth of the study area from 1981 to 2010.

coefficients of correlation of time series and lags of itself [29]. However, the more objective way to choose the orders of $p$ and $q$ of an ARMA (p, q) process is to use objectively defined criteria such as Bayesian Information Criterion (BIC), which is given as formula (8) [30]. The orders of $p$ and $q$ that minimizes $B I C$ value are used as the appropriate orders of the model.

$$
B I C: c_{n}(k)=\frac{k \ln (n)}{n}-\frac{2 \ln (L)}{n},
$$

where $L$ is the likelihood function, $k$ is the number of parameters in the model, and $n$ is the number of observations.

Finally, the diagnostic stage ascertains whether the developed ARIMA model fits well with the input time series data or not. Once it is ensured that the difference between the predicted and actual observations is sufficiently small, then the model can be utilized for forecasting.

2.3. BP Neural Network. BP neural network, called the backpropagation neural network, is a typical type of multilayer feed-forward neural network. It was proposed by Rumelhart and McClelland in 1986, which is one of the most easily understood and widely used neural network algorithms [31, 32]. The structure of the BP neural network is shown in Figure 2. It consists of three layers, including the input layer, output layer, and hidden layer. There is at least one hidden layer. Each layer is composed of several neurons (nodes), via which each layer is connected to its adjacent layer. There is no connection between nodes of the same layer. The input data is stored in the input layer node and transmitted to the output layer through the hidden layer.

The learning and training of the BP neural network adopt the method in which the error function decreases according to the gradient to minimize the mean square error between the actual output value and the expected output value [33]. The learning process is divided into two stages: a multilayer feed-forward stage, which calculates the actual input and output of each node at each layer in turn from the input layer; the backpropagation stage; according to the output error of the output layer neurons, the weight of each connection is corrected along the reverse path to reduce the error [34].

The multilayer feed-forward mathematical model is described by equation (6):

$$
\left\{\begin{array}{l}
y_{i}^{l}=f\left(x_{i}^{l}\right), \\
x_{i}^{l}=\sum_{j=1}^{N_{l-1}} \omega_{i j}^{l} y_{j}^{l-1}+\theta_{i}^{l}, \quad(i=1,2, \ldots, L),
\end{array}\right.
$$

where $y_{i}^{l}$ is the output value for node $i$ of layer $l ; x_{i}^{l}$ is the activation value for node $i$ of layer $l ; w_{i j}^{l}$ is the connection weight for node $j$ of the layer $l-1$ to the node $i$ of the layer $l ; \theta_{i}^{l}$ is the threshold of the node $i$ of the layer $l ; N$ is the number of nodes in the layer; $L$ is the total number of layers; $f()$ is the activation function of the neuron, which is Sigmoid function in this paper described as $f(x)=1 /(1+\mathrm{e}-\mathrm{x})$.

2.4. Coupled Model. In the new coupled model, called the WA-ARMA-BP model, groundwater depth series was decomposed into multiple detailed signal sequences and approximate signal sequences through wavelet decomposition. Then, detailed signal sequences and approximate signal sequences were calculated using the ARMA model and BP neural network model, respectively.

2.5. Model Performance Comparison. In order to test the accuracy of the models, the coefficient of determination $\left(R^{2}\right)$, Nash-Sutcliffe model efficiency coefficient (NSE), and rootmean-squared error (RMSE) were used in this paper.

$R^{2}$ measures the degree of correlation among the observed and modeled values. Its values range from 0 to 1 , with 1 indicating a perfect fit between the data and the line drawn through them. $R^{2}$ is given by [35]

$$
R^{2}=\left(\frac{\sum_{i=1}^{n}\left(O_{i}-\bar{O}\right)\left(M_{i}-\bar{M}\right)}{\sqrt{\sum_{i=1}^{n}\left(O_{i}-\bar{O}\right)^{2}} \sqrt{\sum_{i=1}^{n}\left(M_{i}-\bar{M}\right)^{2}}}\right)^{2},
$$

where $n$ is the number of the observations; $M_{i}$ and $O_{i}$ represent the forecasted and observed values; $\bar{M}$ and $\bar{O}$ represent the mean value of $M_{i}$ and $O_{i}$, respectively.

NSE provides a measure of the ability of a model to predict values that are different from the mean. The values of NSE varies from - $\infty$ to 1 . The closer the value to 1 , the better the model performance. NSE is expressed as [36]

$$
\mathrm{NSE}=1-\frac{\sum_{i=1}^{n}\left(O_{i}-M_{i}\right)^{2}}{\sum_{i=1}^{n}\left(O_{i}-\bar{O}\right)^{2}}
$$


where $n$ is the number of the observations; $M_{i}$ and $O_{i}$ represent the forecasted and observed values; $\bar{O}$ represent the mean value of $O_{i}$.

RMSE indicates the discrepancy between the observed and calculated values. A perfect fit between observed and forecasted values would have an RMSE of 0 . RMSE is expressed as [7]

$$
\mathrm{RMSE}=\sqrt{\frac{\sum_{i=1}^{N}\left(O_{i}-M_{i}\right)^{2}}{N}},
$$

where $n$ is the number of the observations; $M_{i}$ and $O_{i}$ represent the forecasted and observed values.

Leave-one-out cross-validation (LOO-CV) is a commonly used algorithm for estimating the predictive performance of a multivariable calibration model. Usually, practical calibration experiments have to be based on a limited set of available samples. The idea behind the LOO$\mathrm{CV}$ algorithm is to predict the property value for a sample from the data set, which is, in turn, predicted from the calibration model calculated from the data for all other samples. When applying the algorithm to a data set with $N$ examples, the calibration modeling is performed $N$ times, each time using $\mathrm{N}-1$ samples for modeling and one sample for testing. Thus, the procedure of LOO-CV can be divided into $N$ segment. Finally, the $R^{2}$ and RMSE of LOO-CV for the three models can be obtained.

\section{Model Development}

3.1. ARIMA Model. Statistic $U_{M K}{ }^{*}$ of the average groundwater depth series was 1.635 which indicated an increasing trend but not significance. Thus, the average groundwater depth data series was not stationary and it is necessary to transform the data series into a stationary series through the differencing process. After twice differencing processes, it is obvious that both ACF and PACF showed the trailing trend which illustrated that the data series was stationary and it is suitable to build ARIMA (p, 2, q) model for average groundwater depth data series Figure 4. According to the ACF and PACF, the appropriate models were determined as ARIMA (1, 2, 1), ARIMA $(2,2,1)$, ARIMA $(3,2,1)$, ARIMA $(1,2,2)$, ARIMA $(2,2,2)$, and ARIMA $(3,2,2)$. In order to determine the optimal model, the values of BIC were calculated for the above models (Table 2). As shown in Table 2, ARIMA $(3,2,1)$ was the optimal model since the value of $\mathrm{BIC}$ was minimum. Following this, the parameter estimation of the ARMA model was performed. The model was trained using the data from 1981 to 2000 and tested using the data from 2001 to 2010 .

3.2. BP Neural Network. The BP neural network model for average groundwater depth forecasting was developed in MATLAB R2014a software. In this paper, it was considered that the groundwater depth $\left(H_{\mathrm{t}}\right)$ at time $t$ was related to the previous groundwater depth data $\left(H_{t-l}, l=1,2, \ldots\right)$, and the effect of $H_{t-r}$ on $H_{t}$ is greater than that of $H_{t-r-l}$. The groundwater depth data in the previous $n$ years were set as input parameters, and the groundwater depth data of the $(n+1)$ th year were set as output parameters of the BP network model. The number of $n$ was set to 5 . Thus, there were altogether 25 input nodes and 25 output nodes in the BP network model of the study area. The structure of the neural network was determined by trial and error. The optimal number of nodes in the hidden layer and the stopping criteria were optimized by trial and error for obtaining accurate output. The activation function was set as tansig transfer function. Out of the 30-year data sets available, 20 data sets were used for training the BP network model and 10 data sets were used for testing the model.

3.3. Coupled Model. In the coupled WA-ARMA-BP model for average groundwater depth forecasting, the first step was decomposing groundwater depth data series into multiple detailed signal sequences and approximate signal sequences through wavelet decomposition and reconstruction. This work was developed using the wavelet toolbox of MATLAB software. According to the method of wavelet decomposition mentioned above, the groundwater depth series (x) was decomposed threefold as follows:

$$
x=d_{1}+d_{2}+d_{3}+a_{3},
$$

where $d_{1}, d_{2}$, and $d_{3}$ represent the three detailed signal sequences; $a_{3}$ represents the approximate signal sequence (Figure 5).

The three detailed signal sequences were calculated using the ARMA model. In this study, the simulation process is illustrated by taking detailed signal $d_{1}$ as an example. The first step is to estimate the stationarity of the average groundwater depth data via ACF and PACF. As shown in Figure 6, it is obvious that both ACF and PACF showed the trailing trend which illustrated that the $d_{1}$ data series was stationary and it is suitable to build ARMA (p, q) model for detailed signal $d_{1}$ series. Based on the principle of minimizing BIC value, ARMA $(2,1)$ was the optimal model Table 3. Following this, the parameter estimation of the ARMA model was performed. The model was trained using the data from 1981 to 2000 and tested using the data from 2001 to 2010 . The calculation of detailed signals $d_{2}$ and $d_{3}$ followed the procedures above.

The approximate signal sequence was calculated using BP neural network model, which was developed in MATLAB R2014a software. The calculation steps of the model were consistent with the above description. Out of the 30year data sets available, 20 data sets were used for training the BP network model and 10 data sets were used for testing the model.

After obtaining the simulation and prediction models of the above sequences, the models were superposed to achieve the simulation and prediction model of the average groundwater depth.

\section{Results and Discussion}

In order to verify the validity of the proposed prediction method, the ARIMA model and BP neural network model were developed to forecast the groundwater depth directly 

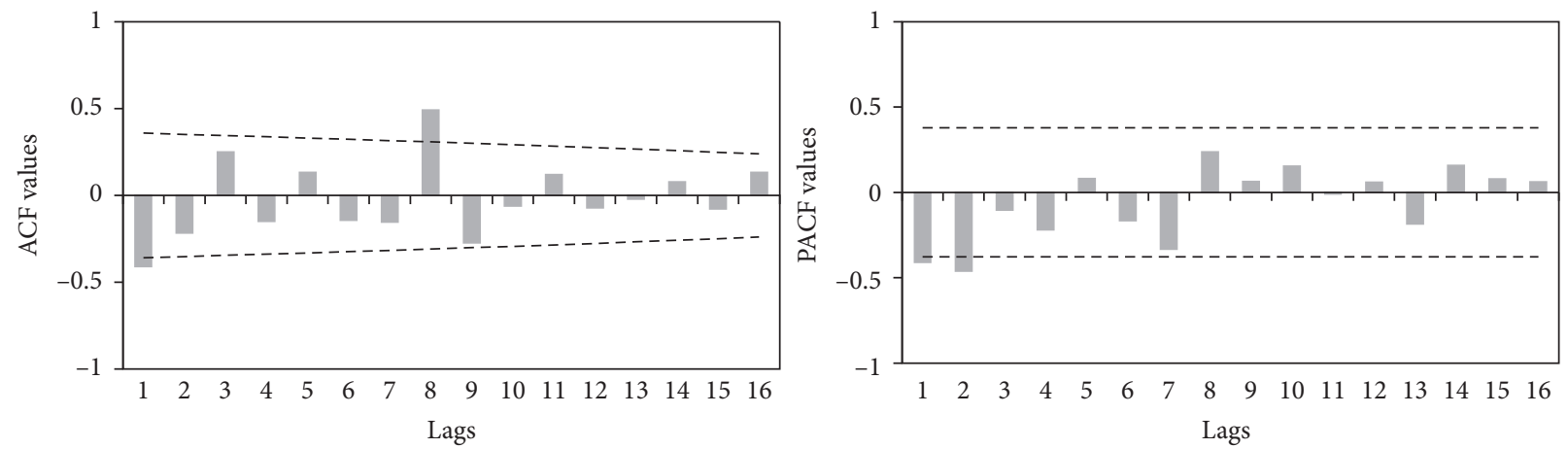

Figure 4: The Auto Correlation Function (ACF) and Partial Auto Correlation Function (PACF) of the average groundwater depth after twice differencing processes.

TABLE 2: The values of BIC of ARIMA models.

\begin{tabular}{lccc}
\hline Model & ARIMA $(1,2,1)$ & ARIMA $(2,2,1)$ & ARIMA (3, 2, 1) \\
\hline BIC & -0.123 & -0.147 & -0.158 \\
Model & ARIMA $(1,2,2)$ & ARIMA $(2,2,2)$ & ARIMA (3, 2, 2) \\
BIC & 0.04 & 0.146 & 0.356 \\
\hline
\end{tabular}
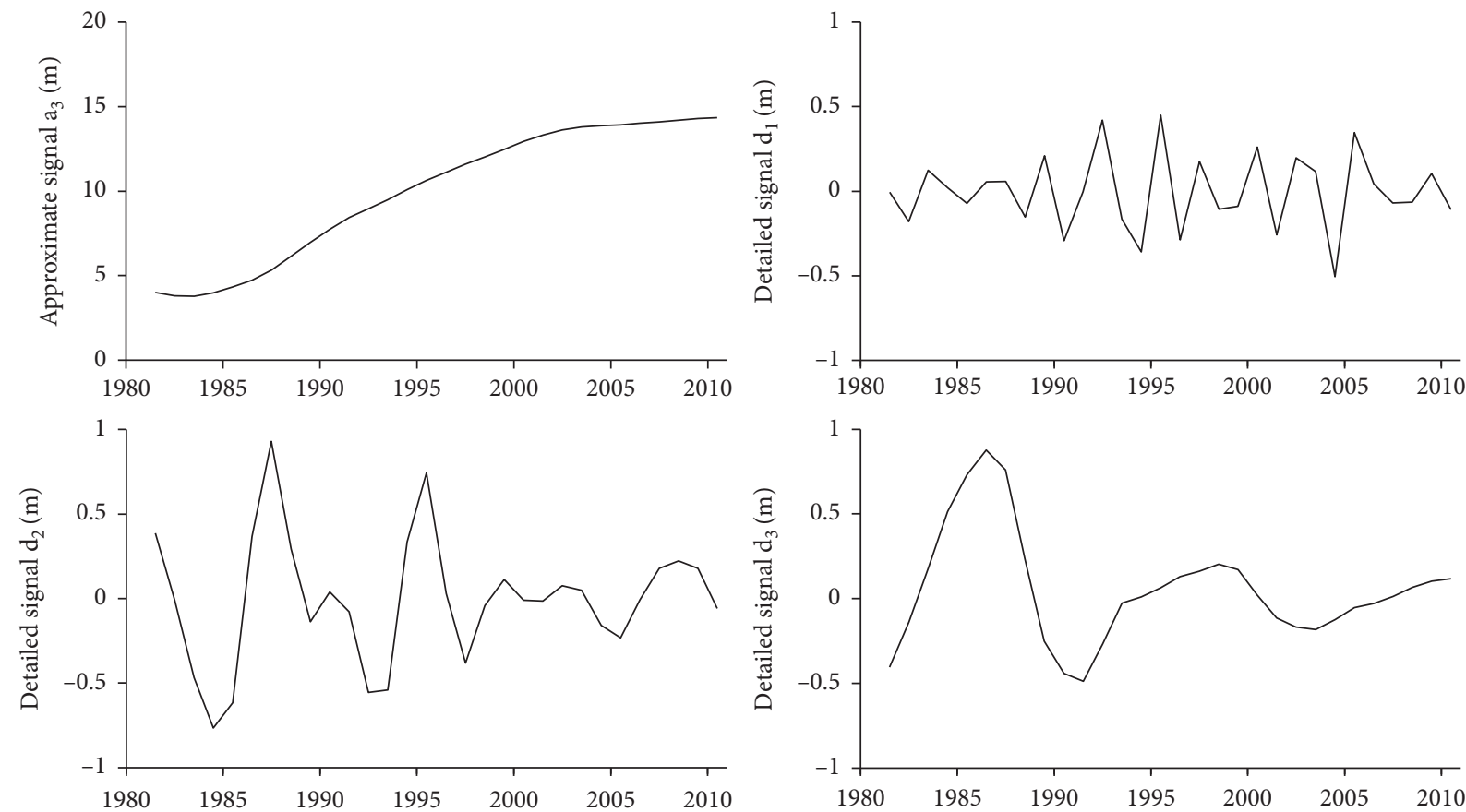

Figure 5: The average groundwater depth series, three detailed signal sequences, and approximate signal sequence through wavelet decomposition and reconstruction.

and then compared with the result of the coupled model. The results of the models are shown in Table 4. The performance statistics of the WA-ARMA-BP model yielded an $R^{2}$ of 0.9572 , an NSE of 0.9482 , and an RMSE of 0.0136 . The performance statistics of the ARIMA model yielded an $R^{2}$ of 0.5731 , an NSE of 0.5607 , and an RMSE of 0.05 . The performance statistics of the ARIMA model obtained an $R^{2}$ of 0.4374 , an NSE of 0.3186 , and an RMSE of 0.0797 . The lower RMSE values (with 0 being a perfect fit value) indicate that the WA-ARMA-BP model had smaller differences and discrepancies between the forecasted average groundwater depth and the observed values. The higher values of $R^{2}$ and NSE (with 1 being a perfect fit value) indicate that the WAARMA-BP model is more accurate. Overall, the WAARMA-BP model performed more efficiently than the ARIMA model and BP neural network model based on $R^{2}$, NSE, and RMSE criteria.

Figure 7 shows a comparison of observed and forecasted average groundwater depth for the test period using the WA-ARMA-BP model, ARIMA model, and BP neural 

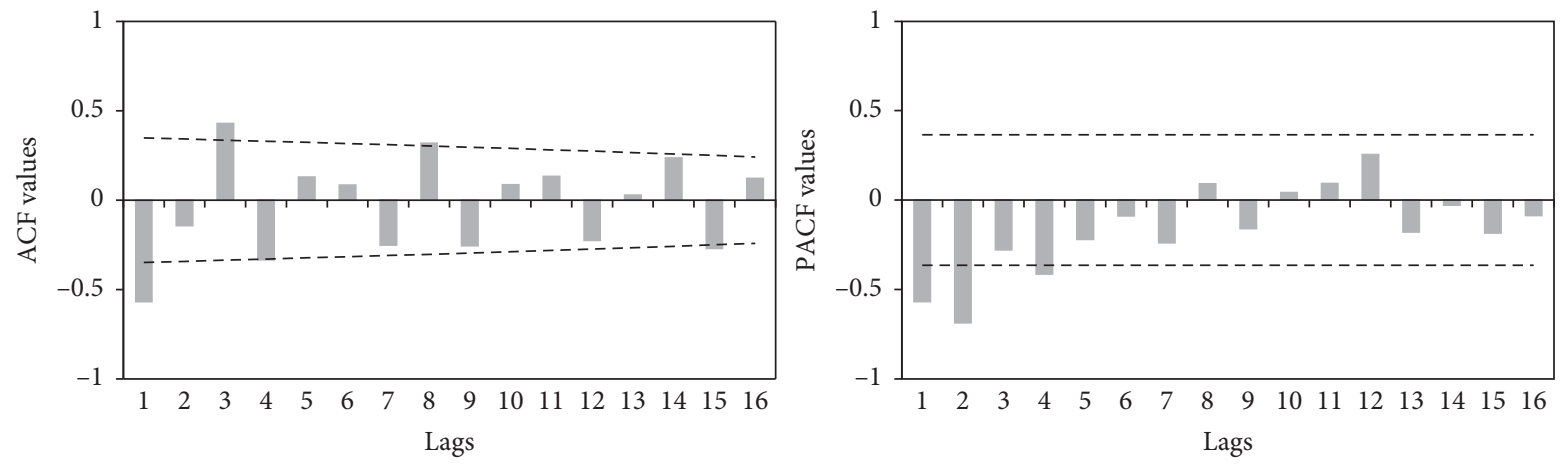

Figure 6: ACF and PACF of the detailed signal $d_{1}$.

TABLE 3: The values of BIC of ARMA models.

\begin{tabular}{lcccc}
\hline Model & ARMA $(1,1)$ & ARMA $(1,2)$ & ARMA $(1,3)$ & ARMA $(2,1)$ \\
\hline BIC & -3.624 & -3.250 & -3.832 & -3.882 \\
Model & ARMA $(2,3)$ & ARMA $(3,1)$ & ARMA $(3,2)$ & ARMA $(3,3)$ \\
BIC & -3.874 & -3.769 & -3.572 & -3.734 \\
\hline
\end{tabular}

TABLE 4: Comparison of the best WA-ARMA-BP model with the best ARIMA model and BP neural network model for average groundwater depth forecasting during the testing period.

\begin{tabular}{lccr}
\hline & WA-ARMA-BP model & ARIMA & BP \\
\hline$R^{2}$ & 0.9534 & 0.5731 & 0.4374 \\
NSE & 0.9482 & 0.5607 & 0.3186 \\
RMSE & 0.0136 & 0.05 & 0.0797 \\
\hline
\end{tabular}

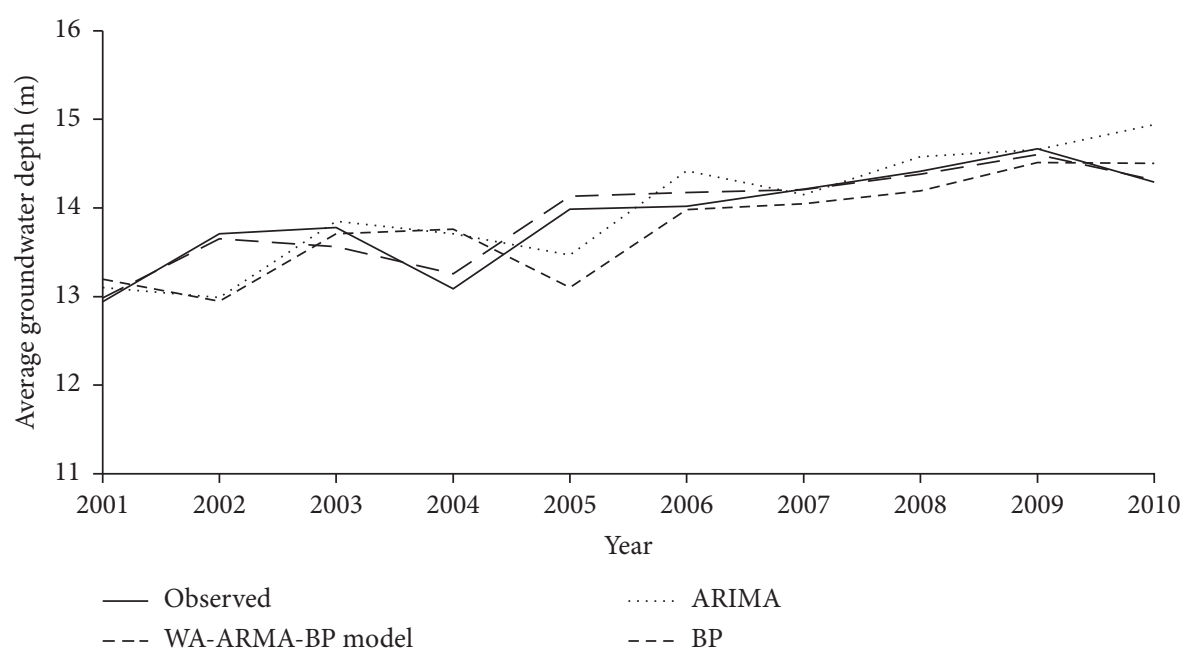

FIGURE 7: Comparison of observed and forecasted average groundwater depth for test period using WA-ARMA-BP model, ARIMA model, and BP neural network model.

network model. It can be seen that the WA-ARMA-BP model provides closer estimates to the corresponding observed groundwater depth than the other two models.

Figures 8-10 are scatterplots comparing the observed and forecasted average groundwater depth using the best WA-ARMA-BP model, ARIMA model, and BP neural network model during the testing period. It can be seen that the WA-ARMA-BP model has less scattered estimates and that the values are denser in the neighborhood of the straight line compared to the ARIMA model and BP neural network model. Overall, it can be concluded that the best WAARMA-BP model provided more accurate forecasting 


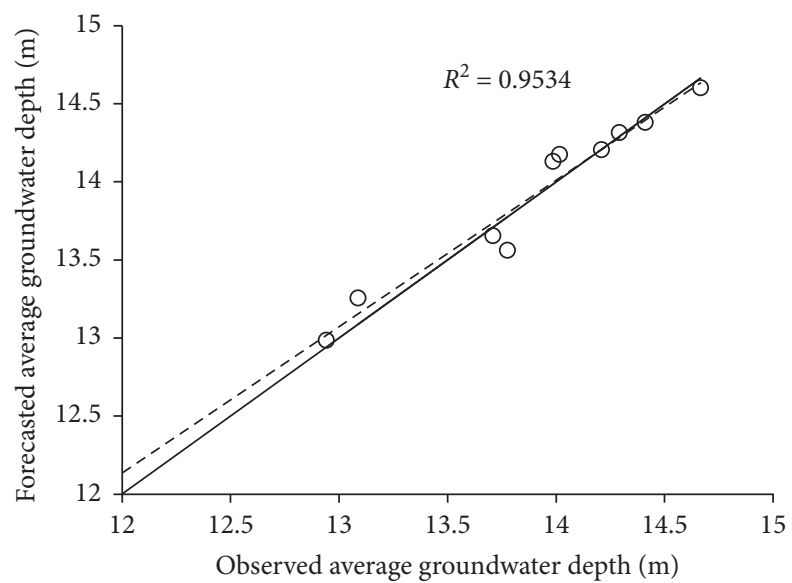

FIGURE 8: Scatterplot comparing observed and forecasted average groundwater depth using the best WA-ARMA-BP model during the testing period.

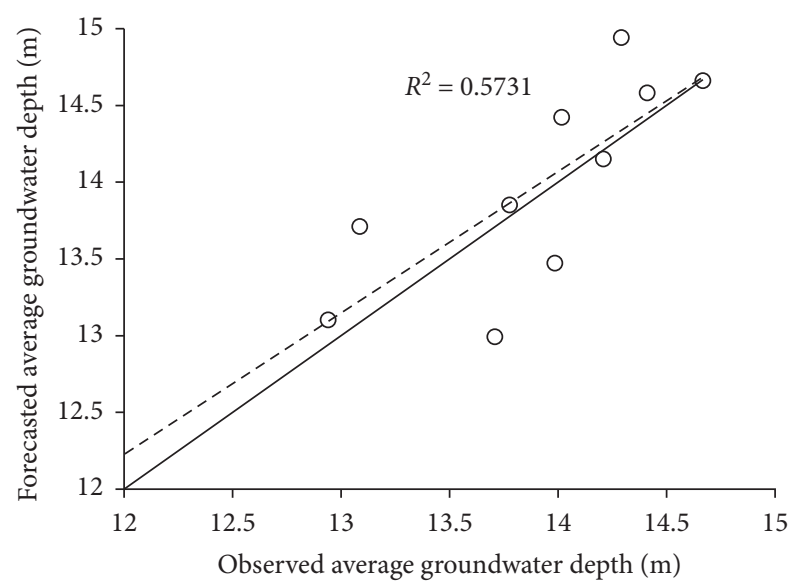

FIGURE 9: Scatterplot comparing observed and forecasted average groundwater depth using the best ARIMA model during the testing period.

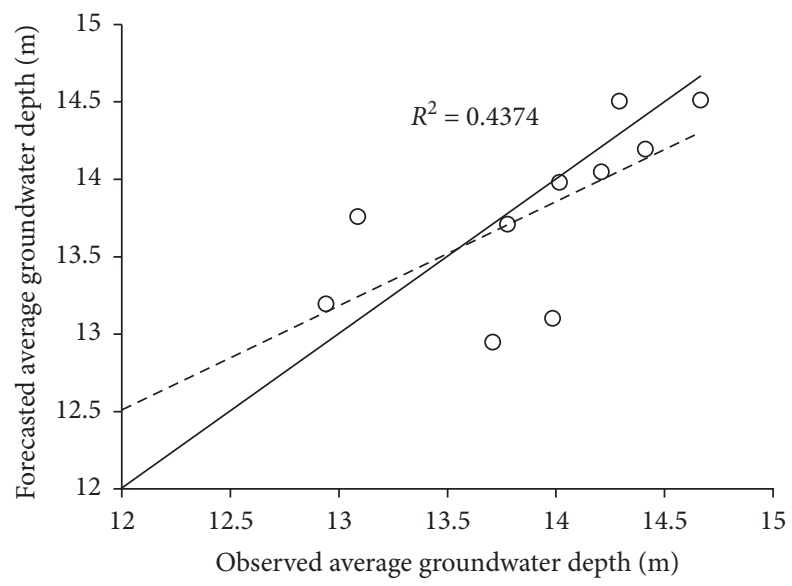

FIGURE 10: Scatterplot comparing observed and forecasted average groundwater depth using the best BP neural network model during the testing period.

TABLE 5: The results of LOO-CV.

\begin{tabular}{lccc}
\hline & WA-ARMA-BP model & ARIMA & BP \\
\hline$R^{2}$ & 0.8954 & 0.5215 & 0.4756 \\
RMSE & 0.0254 & 0.07821 & 0.1034 \\
\hline
\end{tabular}


results than the ARIMA model and BP neural network model for groundwater depth forecasting.

The results of LOO-CV show that the WA-ARMA-BP model has the biggest $R^{2}$ and the smallest RMSE which demonstrates that the model established with WA-ARMA-BP is better than the model established with the ARIMA model and BP neural network model in prediction accuracy (Table 5).

\section{Conclusions}

In this paper, a new method based on coupling wavelet decomposition method (WA), ARMA model, and BP neural network model for groundwater depth forecasting applications was proposed to help manage groundwater supplies in a more effective and sustainable manner. The coupled model (WA-ARMA-BP) was compared to the regular ARIMA model and BP neural network model for average groundwater depth forecasting in Jinghui Canal Irrigation District in the northwest of China. Using the wavelet decomposition method, the original data series was decomposed into three detailed signal sequences which were then forecasted via ARMA models and one approximate signal sequence which was then forecasted via BP neural network. The wavelet decomposition method allowed most of the "noisy" data to be removed and made data changes more stable.

This study found that the WA-ARMA-BP model was substantially more accurate than the ARIMA model and BP neural network model. It is hypothesized that the WAARMA-BP model is more accurate because the wavelet decomposition method allows most of the "noisy" data to be removed and makes data changes more stable. It is indicated that the WA-ARMA-BP model is a potentially very useful new method for nonstationary time series such as groundwater depth forecasting.

\section{Data Availability}

The data can be obtained upon request to the corresponding author.

\section{Conflicts of Interest}

The authors declare that they have no conflicts of interest.

\section{Acknowledgments}

This research was supported by "The Twelfth Five-Year" National Science-Technology Support Plan Projects (2011BAD29B01) and Shaanxi Province Natural Science Foundation of China (2014JM1030).

\section{Supplementary Materials}

All supplementary data including all input parameters and results of these three methods can be found in the supplementary file. (Supplementary Materials)

\section{References}

[1] L. F. Konikow and E. Kendy, "Groundwater depletion: a global problem," Hydrogeology Journal, vol. 13, no. 1, pp. 317-320, 2005.

[2] J. Adamowski and H. F. Chan, "A wavelet neural network conjunction model for groundwater level forecasting," Journal of Hydrology, vol. 407, no. 1-4, pp. 28-40, 2011.

[3] H. Yoon, S.-C. Jun, Y. Hyun, G.-O. Bae, and K.-K. Lee, "A comparative study of artificial neural networks and support vector machines for predicting groundwater levels in a coastal aquifer," Journal of Hydrology, vol. 396, no. 1-2, pp. 128-138, 2011.

[4] A. K. Verma and T. N. Singh, "Prediction of water quality from simple field parameters," Environmental Earth Sciences, vol. 69, no. 3, pp. 821-829, 2013.

[5] N. W. Kim, I. M. Chung, Y. S. Won, and J. G. Arnold, "Development and application of the integrated SWATMODFLOW model," Journal of Hydrology, vol. 356, no. 1-2, pp. 1-16, 2008.

[6] I. Borsi, R. Rossetto, C. Schifani, and M. C. Hill, "Modeling unsaturated zone flow and runoff processes by integrating MODFLOW-LGR and VSF, and creating the new CFL package," Journal of Hydrology, vol. 488, pp. 33-47, 2013.

[7] R. Barzegar, E. Fijani, A. Asghari Moghaddam, and E. Tziritis, "Forecasting of groundwater level fluctuations using ensemble hybrid multi-wavelet neural network-based models," Science of The Total Environment, vol. 599-600, pp. 20-31, 2017.

[8] Q. Yang, Z. Hou, Y. Wang, Y. Zhao, and J. Delgado, “A comparative study of shallow groundwater level simulation with WA-ANN and ITS model in a coastal island of south China," Arabian Journal of Geosciences, vol. 8, no. 9, pp. 6583-6593, 2014.

[9] I. N. Daliakopoulos, P. Coulibaly, and I. K. Tsanis, "Groundwater level forecasting using artificial neural networks," Journal of Hydrology, vol. 309, no. 1-4, pp. 229-240, 2005.

[10] V. J. Bidwell, "Realistic forecasting of groundwater level, based on the eigenstructure of aquifer dynamics," Mathematics and Computers in Simulation, vol. 69, no. 1-2, pp. 12-20, 2005.

[11] S.-I. Lee, S.-K. Lee, and S.-Y. Hamm, "A model for groundwater time-series from the well field of riverbank filtration," Journal of Korea Water Resources Association, vol. 42, no. 8, pp. 673-680, 2009.

[12] S. M. Guzman, J. O. Paz, and M. L. M. Tagert, "The use of NARX neural networks to forecast daily groundwater levels," Water Resources Management, vol. 31, no. 5, pp. 1591-1603, 2017.

[13] Z. M. Yaseen, W. H. M. W. Mohtar, A. M. S. Ameen et al., "Implementation of univariate paradigm for streamflow simulation using hybrid data-driven model: case study in tropical region," IEEE Access, vol. 7, pp. 74471-74481, 2019.

[14] B. Cannas, A. Fanni, L. See, and G. Sias, "Data preprocessing for river flow forecasting using neural networks: wavelet transforms and data partitioning," Physics and Chemistry of the Earth, Parts $A / B / C$, vol. 31, no. 18, pp. 1164-1171, 2006.

[15] T. Partal and O. Kisi, "Wavelet and neuro-fuzzy conjunction model for precipitation forecasting," J. Hydrol, vol. 342, no. 12, pp. 199-212, 2007.

[16] J. Adamowski, "Development of a short-term river flood forecasting method based on wavelet analysis," J. Hydrol, vol. 353, no. 3-4, pp. 247-266, 2007.

[17] M. K. Tiwari and C. Chatterjee, "Development of an accurate and reliable hourly flood forecasting model using wavelet- 
bootstrap-ANN (WBANN) hybrid approach," Journal of Hydrology, vol. 394, no. 3-4, pp. 458-470, 2010.

[18] W. Wang, J. Jin, and Y. Li, "Prediction of inflow at three Gorges Dam in Yangtze river with wavelet network model," Water Resources Management, vol. 23, no. 13, pp. 2791-2803, 2009.

[19] L. Yang, Ecological Environment Problems Analysis and Regulation Syudy in the Development of Jinghui Canal Irrigation District in Shaanxi Province, Xi'an University of Technology, Xi'an, China, 2017.

[20] S. G. Mallat, "Multifrequency channel decompositions of images and wavelet models," IEEE Transactions on Acoustics, Speech, and Signal Processing, vol. 37, no. 12, pp. 2091-2110, 1989.

[21] S. Yue, P. Pilon, B. Phinney, and G. Cavadias, "The influence of autocorrelation on the ability to detect trend in hydrological series," Hydrological Processes, vol. 16, no. 9, pp. 1807-1829, 2002.

[22] K. H. Hame and A. R. Rao, "A modified Mann-Kendall trend test for autocorrelated data," Journal of Hydrology, vol. 204, pp. 182-196, 1998.

[23] K. H. Hame, "Trend detection on hydrologic data: the MannKendall trend test under the scaling hypothesis," Journal of Hydrology, vol. 349, pp. 350-363, 2008.

[24] M. N. Khaliq, T. B. M. J. Ouardat, and P. Gachon, "Identification of temporal trends in annual and seasonal low flows occurring in Canadian rivers: The effect of short- and longTerm persistence," Journal of Hydrology, vol. 369, pp. 183-197, 2009.

[25] H. B. Mann, "Non-parametric tests against trend," Econometrica, vol. 13, pp. 245-259, 1945.

[26] M. G. Kendall, Rank auto-correlation methods, Charles Griffin, London, England, 1975.

[27] R. H. Shummway and D. S. Stoffer, Time Series Analysis and its Application with R Examples, Springer, Pittsburg, 2006.

[28] K. Soni, S. Kapoor, K. S. Parmar, and D. G. Kaskaoutis, "Statistical analysis of aerosols over the Indo-Gangetic plains and southern slopes of the Himalayas using ARIMA model based on long-term MODIS observations," Atmospheric Research, vol. 14, pp. 174-192, 2004.

[29] G. E. P. Box, G. M. Jenkins, and G. C. Reinsel, "Time series analysis: forecasting and control," Journal of Marketing Research, vol. 14, 2008.

[30] G. Schwarz, "Estimating the dimension of a model," The Annals of Statistics, vol. 6, no. 2, pp. 461-464, 1978.

[31] D. E. Rumelhart, G. E. Hinton, and R. J. Williams, Learning Internal Representation by Error Propagation, Neurocomputing: Foundations of Research, MIT Press, Cambridge, England, 1988.

[32] C. A. L. Bailer-Jones, R. Gupta, H. P. Singh, and A. B. Bulsari, "An introduction to artifcial neural networks," Neural Networks for Chemical Engineers, vol. 19, pp. 36-62, 2001.

[33] E. A. Shams and A. Rizaner, "A novel support vector machine based intrusion detection system for mobile ad hoc networks," Wireless Networks, vol. 24, no. 5, pp. 1821-1829, 2018.

[34] W. Li, G. Xu, Q. Xing, and M. Lyu, "Application of improved AHP-BP neural network in CSR performance evaluation model," Wireless Personal Communications, vol. 111, no. 4, pp. 2215-2230, 2019.

[35] I. Pulido-Calvo and J. C. Gutiérrez-Estrada, "Improved irrigation water demand forecasting using a soft-computing hybrid model," Biosystems Engineering, vol. 102, no. 2, pp. 202-218, 2009.
[36] P. Sreekanth, D. N. Geethanjali, P. D. Sreedevi, S. Ahmed, N. R. Kumar, and P. D. K. Jayanthi, "Forecasting groundwater level using artificial neural networks," Current Science, vol. 96, pp. 933-939, 2009. 